\title{
Errata: Hydrodynamic Theory of Electron Transport in a Strong Magnetic Field ${ }^{1}$
}

\author{
M. C. Marchetti, T. R. Kirkpatrick, and J. R. Dorfman
}

Received June 8, 1987

The following errors are to be corrected:

1. In Eq. $(2.15 \mathrm{c})$ the minus sign should be replaced by a plus sign.

2. Eq. (2.16) should read

$$
\Omega_{i j}(\mathbf{k})=\left(a_{i,-\mathbf{k}}, \hat{\mathbf{k}} \cdot \mathbf{j}_{\hat{\mathbf{k}}}^{j}\right)\left(a_{j,-\mathbf{k}}, a_{j, \mathbf{k}}\right)^{-1}
$$

3. Eq. (2.19b) should read

$$
I_{\alpha, \mathbf{k}}^{5}=\left[j_{\alpha, \mathbf{k}}^{\varepsilon}-(h / \rho) j_{\alpha, \mathbf{k}}^{\rho}\right] / \rho C_{v}
$$

4. In Eqs. (2.21a)-(2.21e) the second integral sign should be deleted.

5. In Eqs. (2.22a) and (2.22b) the factor in front of the integral should be $\rho C_{v} / k_{\mathrm{B}} T^{2}$, not $1 /\left(\rho C_{v} k_{\mathrm{B}} T^{2}\right)$.

6. Some terms were left out of Eq. (A.4a). For clarity we rewrite here the entire corrected equation. It should be noted that the correct form was used in the calculation.

$$
\begin{aligned}
z_{\mu}^{(2)}(k)= & \left.\frac{z_{\mu}^{(0)}}{2\left[2\left(z_{\mu}^{(0)}\right)^{2}+\omega_{h}^{2}\right.}\right]\left\{-\frac{\gamma}{\rho \chi_{T}} k^{2}\left[1+\frac{\omega_{\mathrm{B}}^{2}}{\left(z_{\mu}^{(0)}\right)^{2}} \hat{k}_{z}^{2}\right]\right. \\
& \left.+2 \omega_{\mathrm{B}}\left(1+\frac{\omega_{p}^{2}}{\left(z_{\mu}^{(0)}\right)^{2}} \hat{k}_{z}^{2}\right)\left(v_{3 \mu} k_{\perp}^{2}+v_{4 \mu} k_{z}^{2}\right)\right\} \\
& +\frac{1}{2\left[2\left(z_{\mu}^{(0)}\right)^{2}+\omega_{h}^{2}\right]}\left\{\left(z_{\mu}^{(0)}\right)^{2}\left[v_{2 \mu} k^{2}+k_{\perp}^{2}\left(v_{1 \mu}+\frac{v_{0 \mu}}{3}\right)+\frac{4}{3} v_{0 \mu} k_{z}^{2}\right]\right.
\end{aligned}
$$

\footnotetext{
${ }^{1}$ This paper appeared in J. Stat. Phys. 46:679 (1987).
} 


$$
\begin{aligned}
& +\omega_{p}^{2} \hat{k}_{z}^{2} k_{\perp}^{2}\left(v_{1 \mu}+3 v_{0 \mu}-\frac{2 \omega_{\mathrm{B}}}{z_{\mu}^{(0)}} v_{4 \mu}-4 v_{2 \mu}\right) \\
& -\omega_{\mathrm{B}}^{2}\left(1+\frac{\omega_{p}^{2}}{\left(z_{\mu}^{(0)}\right)^{2}} \hat{k}_{z}^{2}\right)\left(v_{1 \mu} k_{\perp}^{2}+v_{2 \mu} k_{z}^{2}\right) \\
& \left.+\omega_{p}^{2} v_{2 \mu} k^{2}+\omega_{\mathrm{B}}^{2}\left(v_{2 \mu} k_{\perp}^{2}+\frac{4}{3} v_{0 \mu} k_{z}^{2}\right)\right\}
\end{aligned}
$$

We thank A. J. Schoolderman and L. G. Suttorp for pointing out some of these errors. The hydrodynamic modes for a classical electron gas in a magnetic field can also be found in L. G. Suttorp and A. J. Schoolderman, Physica 141A:1 (1987). 